\title{
The potential contribution of tumour-related factors to the development of FOLFOX-induced sinusoidal obstruction syndrome
}

\author{
S M Robinson ${ }^{* 1,2}$, D A Mann ${ }^{2}$, D M Manas ${ }^{1}$, F Oakley $^{2}$, J Mann ${ }^{2,3}$ and S A White ${ }^{1,2,3}$ \\ ${ }^{1}$ Department of HPB Surgery, Freeman Hospital, Newcastle upon Tyne, NE7 7DN, UK and ${ }^{2}$ Institute of Cellular Medicine, \\ Newcastle University, Newcastle upon Tyne, NE2 4HH, UK
}

Background: Chemotherapy-associated liver injury (CALI) has been linked to increased morbidity and poorer disease-specific outcomes in patients undergoing resection of colorectal liver metastases (CRLM). The aim of this study was to assess the contribution of tumour-related factors to the development of FOLFOX-induced liver injury.

Methods: We assessed the effect of FOLFOX treatment on the murine liver either in the presence or absence of CRLM to evaluate the contribution of both chemotherapy and tumour death to the development of CALI.

Results: In the presence of liver metastases, there was increased hepatic expression of plasminogen activator inhibitor-1 (146-fold; $P<0.01)$ and VWF $(2.4$-fold; $P<0.01)$ transcript as compared with sham-operated controls. In addition, we detected large clusters of megakaryocytes in the spleen of FOLFOX-treated tumour-bearing animals. The livers of FOLFOX-treated animals also showed changes in matrix remodelling genes such as TGF $\beta(P<0.01)$, MMP2 $(P<0.001)$, TIMP1 $(P<0.001)$ and Pro-Collagen I $(P<0.05)$ which was exacerbated in the presence of tumour. These genes have previously been demonstrated to have a key role in FOLFOX-induced liver injury.

Conclusion: It appears that the toxicity of FOLFOX chemotherapy is enhanced by tumour-related factors.

Colorectal cancer is the second leading cause of cancer-related death in the western world. Up to $50 \%$ of patients with colorectal cancer will develop liver metastases and in $25 \%$ of patients are present at the time of primary diagnosis (Stangl et al, 1994). Resection is the gold standard treatment for colorectal liver metastases (CRLM) with 5-year survival in the order of 50-60\% being achievable (Rees et al, 2008). Unfortunately a significant proportion of patients are not suitable for surgery at the time of presentation and require neoadjuvant chemotherapy with the aim of down-staging their disease such that they become operable. In those patients successfully down-staged long-term survival can be achieved which is comparable with those patients offered surgery from the outset (Alberts et al, 2005).
The most commonly used chemotherapy for CRLM consists of folinic acid/5-FU (or the oral pro-drug Capecitabine) in combination with either Oxaliplatin (FOLFOX) or Irinotecan (FOLFIRI) both of which have widely proven efficacy in down-staging CRLM (Robinson et al, 2009). Nonetheless these regimens are associated with the development of chemotherapy-associated liver injury (CALI). The nature of the injury appears to be regimen specific with FOLFIRI being associated with steatohepatitis, whereas FOLFOX is linked to the development of sinusoidal obstruction syndrome (SOS) and nodular regenerative hyperplasia (Robinson et al, 2009; Rubbia-Brandt et al, 2010). The presence of any underlying hepatic parenchymal disease increases the risk of postoperative hepatic insufficiency and as such the presence of CALI is

*Correspondence: SM Robinson; E-mail: s.m.robinson@ncl.ac.uk

This work has been previously presented, in part, at the AUGIS 2011 Annual Scientific Meeting and the Society of Academic and Research Surgery Annual Meeting 2012.

${ }^{3}$ These authors contributed equally to this work

Received 10 July 2013; revised 28 August 2013; accepted 12 September 2013;

published online 10 October 2013

(c) 2013 Cancer Research UK. All rights reserved 0007 - 0920/13

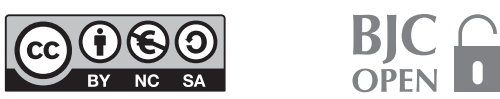


a cause of major concern when undertaking more extensive resections (de Meijer et al, 2010). In addition, CALI has been linked to poorer long-term disease-specific outcomes (Tamandl et al, 2011).

Increasingly an ever more aggressive approach is being taken to the management of CRLM with increasing numbers of patients undergoing second and third resections for recurrent disease (Adam et al, 1997; Adam et al, 2003). Frequently these procedures are interspersed with repeated cycles of chemotherapy meaning that CALI is becoming an ever more pressing issue facing the surgeon managing this cohort of patients. Understanding the pathogenesis of this condition would enable the identification of therapeutic strategies to reduce the incidence of this complication of chemotherapy and thereby improve the safety of surgery in this cohort of patients.

Several authors have attempted to investigate the pathogenesis of this condition using human tissue obtained at the time of liver resection (Pilgrim et al, 2011; Rubbia-Brandt et al, 2011; Agostini et al, 2012). The limitation of this approach is that although it provides an understanding of the ultimate effect of chemotherapy administration on the liver, it does not enable one to investigate the biological mechanisms resulting in these changes or how their manipulation may impact on subsequent liver injury. To do this, a realistic in vivo experimental model is required which reflects, as closely as possible, the events occurring in patients. To this end, we recently developed a murine model of chemotherapy-induced liver injury which enabled us to identify a number of the key pathological processes involved in the pathogenesis of Oxaliplatininduced SOS (Robinson et al, 2013).

Data from two cohort studies have suggested that tumourrelated factors may contribute to the development of SOS (Soubrane et al, 2010; Tamandl et al, 2011). The aim of this study was to determine what impact tumour-related factors might have on Oxaliplatin-induced SOS.

\section{MATERIALS AND METHODS}

All animal studies were subject to review and approval by the local research ethics committee and the UK home office. The use of human tissue for research was approved by the NHS research ethics committee (reference 11/NE/0009 \& 07/Q0906/15) and informed consent was obtained from all participants.

In vitro chemotherapy model. The MCA38 colorectal cancer cell line was a gift from Dr Mario Paolo Colombo (Istitutio Tumori, Milan, Italy). Cells were cultured in Dulbecco's modified eagle medium supplemented with $10 \%$ fetal calf serum, $100 \mathrm{Uml}^{-1}$ Penicillin, $100 \mu \mathrm{g} \mathrm{ml}^{-1}$ Streptomycin and $2 \mathrm{mM}$ L-Glutamine.

The $\mathrm{IC}_{50}$ concentration of Oxaliplatin $(7 \mu \mathrm{M})$ and 5-FU $(14 \mu \mathrm{M})$ at $48 \mathrm{~h}$ were determined by MTT assay. When treating MCA38 cells with FOLFOX, they were seeded and allowed to adhere overnight before exchanging media for that supplemented with $10 \mu \mathrm{M}$ folinic acid. Oxaliplatin was then added followed by $5-\mathrm{FU}$ $2 \mathrm{~h}$ later (Fischel et al, 2002).

Hepatic tumour implantation. $\mathrm{C} 57 \mathrm{Bl} / 6$ mice were subject to laparotomy under isoflurane anaesthesia. In all, $1 \times 10^{5}$ MCA38 cells stably transfected with the pGL4.51 luciferase reporting vector (Promega, Southampton, UK) were then injected beneath the capsule of the left lobe of the liver in $10 \mu \mathrm{l}$ of Hanks buffered salt solution. Haemostasis was achieved by manual compression and the injection track sealed with butylcyanoacrylate glue (Indermil, Henkel Ltd, Hatfield, UK). Animals were allowed to recover for 5 days before the administration of chemotherapy. Sham-operated controls underwent laparotomy only.

The FOLFOX chemotherapy regimen consisted of weekly cycles of intraperitoneal (i.p.) FOLFOX consisting of $6 \mathrm{mg} \mathrm{kg}^{-1}$
Oxaliplatin followed $2 \mathrm{~h}$ later by $50 \mathrm{mg} \mathrm{kg}^{-1} 5$-FU and $90 \mathrm{mg} \mathrm{kg}^{-1}$ folinic acid. This drug-dosing schedule was based upon a careful review of the literature and has been previously used within our unit to induce histological changes of SOS in C57BL/6 J mice after 5 weeks of treatment. Control animals were treated with vehicle alone (Boughattas et al, 1989; Cao et al, 1996; Keizman et al, 2010; Robinson et al, 2013).

Tumour-bearing mice and sham-operated controls were treated with IP FOLFOX, or vehicle control, giving a total of four groups of animals ( $n=6$ per group). The treatment protocol had to be terminated one day after the third treatment owing to large tumour size in the control animals and significant weight loss in the FOLFOX-treated group.

Tumour size in culled animals was assessed using the tumour to body weight ratio (tumour weight/pre-mortem body weight) and by estimating the tumour volume (tumour width ${ }^{2} \times$ tumour length $\times 0.5$ ).

Patient tissue collection. A $1-\mathrm{cm}^{3}$ segment of non-tumourbearing liver tissue was collected from patients undergoing elective liver resection and stored in RNA later (Sigma-Aldrich, Dorset, UK) prior to storage at $-80^{\circ} \mathrm{C}$. Biopsies were taken immediately after opening the abdomen prior to commencing parenchymal transaction or vascular inflow occlusion. Clinical data including the use of pre-operative chemotherapy were recorded for each patient. Histology reports from these patients were reviewed to identify those with evidence of vascular injury to the hepatic parenchyma in keeping with a diagnosis of SOS. Patients with underlying chronic liver disease or primary liver tumours were excluded from analysis.

Three groups of patients were identified for subsequent analysis - those who were chemotherapy naive $(n=10)$; those who received pre-operative Oxaliplatin-based chemotherapy but had no evidence of vascular injury $(n=9)$ and those who received pre-operative chemotherapy and had an identifiable vascular injury $(n=13)$.

RNA extraction, cDNA synthesis and qRT-PCR. Total RNA was extracted using the RNeasy purification system (Qiagen, Manchester, $\mathrm{UK})$. One microgram of total RNA was used to generate cDNA using MMLV-RT and a random hexamer primer (Promega). Quantitative real-time PCR (qRT-PCR) was performed using SYBR-green reagents (Sigma-Aldrich). GAPDH was used as the internal control.

Immunohistochemistry. Sections were deparaffinised and endogenous peroxidase activity blocked. Following antigen retrieval, non-specific binding was inhibited by using the avidin/biotin blocking kit (Vector Laboratories, Burlingame, CA, USA) followed by incubation for $30 \mathrm{~min}$ with $20 \%$ swine serum. Primary antibodies were added and sections incubated overnight at $4{ }^{\circ} \mathrm{C}$. The following morning sections were washed then incubated with an appropriate biotin-conjugated secondary antibody for $2 \mathrm{~h}$ then washed and further incubated for $1 \mathrm{~h}$ with Streptavidin BiotinPeroxidase Complex (Vector Laboratories). Positive cells were visualised with 3,3'-diaminobenzidine tetrahydrochloride (DAB) (Vector Laboratories) and counterstained with haematoxylin. Sections were blinded and the number of positive cells was counted in 15 high-power fields (HPFs).

CXCL1 ELISA/extracellular ATP assay cell culture supernatants. Supernatant was harvested from MCA38 cells treated with FOLFOX chemotherapy, or vehicle control, for $24 \mathrm{~h}$ and snap frozen at $-80^{\circ} \mathrm{C}$. CXCL1 was measured in the cell culture supernatants using a CXCL1 DuoSet ELISA Development Kit (R\&D Systems, Abingdon, UK).

Statistical analysis. Numerical data are expressed as mean value \pm s.e.m. Statistical significance was assessed using 
Mann-Whitney $U$-test and Kruskall-Wallis one-way analysis of variance as appropriate for in vivo data. A two-tailed Student's $t$-test was used for in vitro $\mathrm{qRT}$-PCR data. A $P$-value of $<0.05$ was considered to be statistically significant.

\section{RESULTS}

FOLFOX treatment of colorectal tumour cells activates inflammatory signalling. Recent reports have suggested that cancer cell death by Oxaliplatin-induced apoptosis may not be an immunologically silent event but rather may result in the local release of a variety of pro-inflammatory mediators (Tesniere et al, 2008). To explore whether these tumour-produced mediators might contribute to Oxaliplatin-induced liver injury, it was decided to establish experimental CRLM in C57/Bl6 mice with the syngeneic MCA38 colorectal cancer cell line.

Prior to in vivo studies, death of MCA38 cells in response to FOLFOX was confirmed in vitro (Figure 1A). In addition, it was observed that FOLFOX-induced expression of chemokines associated with inflammation and wound-repair responses (Figure 1B). A greater than 30 -fold increase in CXCL1 transcript (the murine equivalent of IL-8) was confirmed by measuring a three-fold increase in secretion of soluble CXCL1 protein (Figure 1C). CXCL5, CCL2 and CCL5 chemokines were also induced by FOLFOX indicative of cell-damage-associated activation of inflammatory gene expression in the culture.
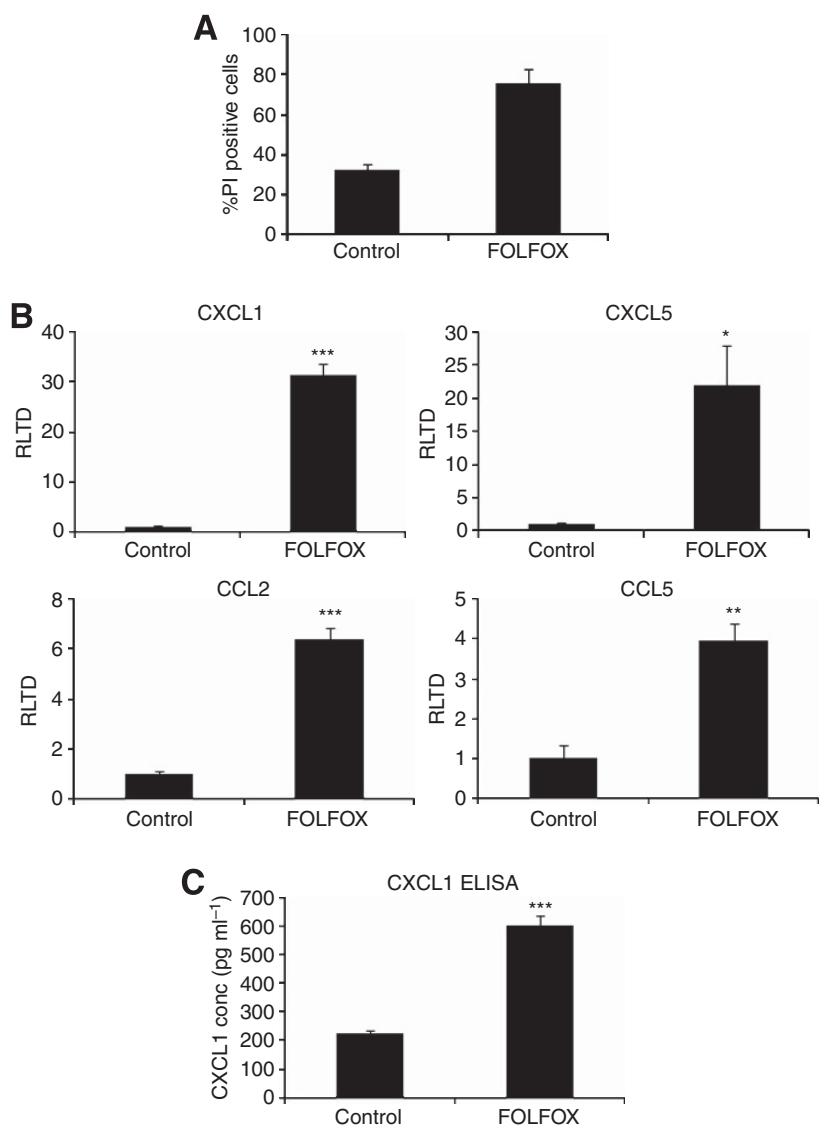

Figure 1. MCA38 cells were treated in vitro with FOLFOX. Cell death in response to FOLFOX after $48 \mathrm{~h}$ treatment was confirmed by propidium iodide staining (A). MCA38 cells treated with FOLFOX for $24 \mathrm{~h}$ show increased expression of the chemokines CXCL1, CXCL5, CCL2 and CCL5 (B). The increased expression of CXCL1 transcript was confirmed by ELISA (C). $\left.{ }^{*}=P<0.05,{ }^{\star *}=P<0.01,{ }^{\star \star *}=P<0.001\right)$.
We have previously reported that increased hepatic expression of these chemokines appears to be implicated in the pathogenesis of SOS in FOLFOX-treated C57BL/6J mice (Robinson et al, 2013). It may be that their production of by apoptosing colorectal cancer cells within the liver contributes further to this process and it was elected to further test this hypothesis in vivo.

Combined tumour and FOLFOX therapy stimulates matrix remodelling. Preliminary experiments demonstrated that 5 days following hepatic implantation of $10^{5}$ MCA-38 cells, a solid tumour was detectable by IVIS imaging (Figure 2A) and on this basis this was the time point at which treatment with FOLFOX, or vehicle control, was commenced. The in vivo effectiveness of this chemotherapy regimen was demonstrated by a marked reduction in the tumour volume (Figure $2 \mathrm{~B} ; P=0.063$ ) and tumour to body weight ratio (Figure 2C; $P=0.041$ ) in FOLFOX-treated animals. When untreated these tumours produced a large local mass arising from the liver parenchyma (Figure 2D and E) and in some animals lung metastases were detectable although this was a late event. Significant weight loss in both FOLFOX groups indicated systemic toxicity of the chemotherapy regimen (Figure $2 \mathrm{~F}$ ).

Neither H\&E (Figure 2G) nor Reticulin (not shown)-stained sections of the liver demonstrated sufficient changes to fit the diagnostic criteria for SOS. We have previously demonstrated that 5 weeks of FOLFOX treatment is necessary in C57BL/6J mice to induce histological changes of SOS and the absence of positive findings in this regard is not surprising (Robinson et al, 2013). Nevertheless, we elected to interrogate this model further to determine if molecular changes within the liver implicated in the pathogenesis of SOS were enhanced by the presence of tumour.

Matrix remodelling and the subsequent development of sinusoidal fibrosis is a cardinal feature of SOS. Hepatic stellate cells (HSCs) have pivotal roles in matrix remodelling, particularly in hepatic fibrogenesis. During this process, quiescent HSCs become activated whereby they take on a myofibroblast phenotype and express smooth muscle $\alpha$-actin ( $\alpha$ SMA) and procollagen I. Figure $3 \mathrm{~A}$ shows that FOLFOX treatment of tumour-bearing mice results in up-regulation of $\alpha$ SMA $(P=0.082)$ and procollagen I $(P=0.009)$ transcripts when compared with sham-operated vehicle controls, in a manner similar to that seen with mice which developed the histological changes of SOS (Robinson et al, 2013). This fibrogenic state was supported by a modest increase in numbers of hepatic $\alpha \mathrm{SMA}+$ cells (7.1 vs 9.9 cells per HPF; $P=0.015)$ as detected by immunohistochemistry (Figure $3 \mathrm{~B}$ ). In order to confirm the relevance of increased $\alpha \mathrm{SMA}$ in the pathogenesis of Oxaliplatin-induced SOS, we performed qRT-PCR on mRNA isolated from the non-tumour-bearing liver of patients undergoing resection of CRLM. In those patients with Oxaliplatininduced vascular injury, we were able to demonstrate a significant increase in expression of $\alpha \mathrm{SMA}$ as compared with those receiving Oxaliplatin who do not develop a vascular injury $(P=0.019$; Figure 3C).

A similar pattern was seen in the hepatic expression of tissue inhibitor of metalloproteinases 1 (TIMP 1) transcript in tumour-bearing FOLFOX-treated mice (Figure 3D; $P=0.004$ ) again in keeping with the process of matrix remodelling in SOS that we have previously reported. It appears that this response is, in part, driven by the master regulator TGF $\beta 1$, the transcript levels of which are maximally elevated in tumour-bearing animals treated with FOLFOX (Figure 3E; $P=0.004$ ). FOLFOX treatment resulted in a 13 -fold up-regulation of MMP2 in tumour-bearing animals treated with FOLFOX $(P<0.002)$, whereas no change in expression was detectable in shamoperated animals (Figure 3F).

Previous published data both from experimental animal studies and human microarray studies have suggested that FOLFOXinduced liver injury is associated with increased hepatic expression 


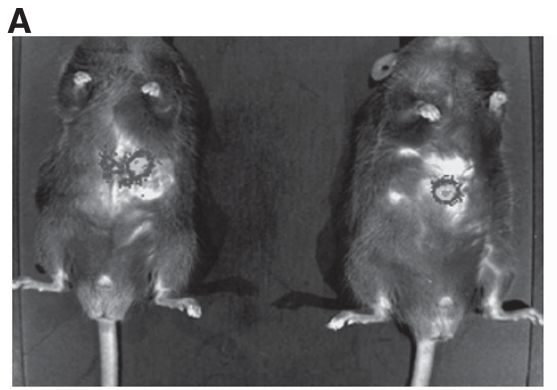

B

C

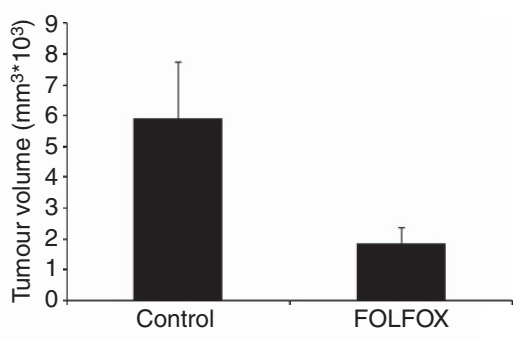

\section{D}
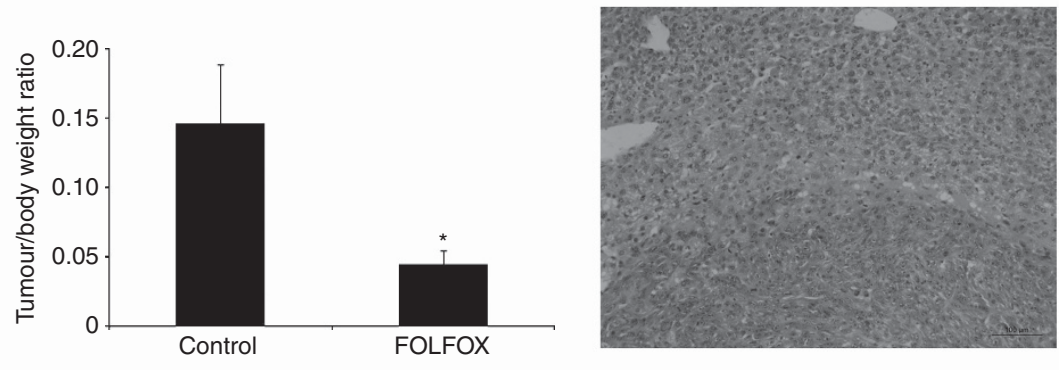

E

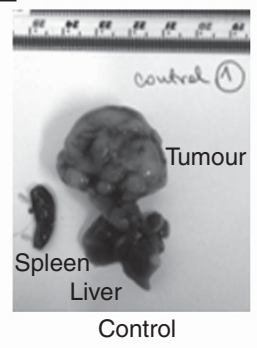

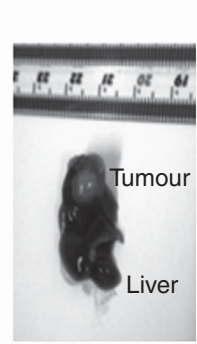

FOLFOX

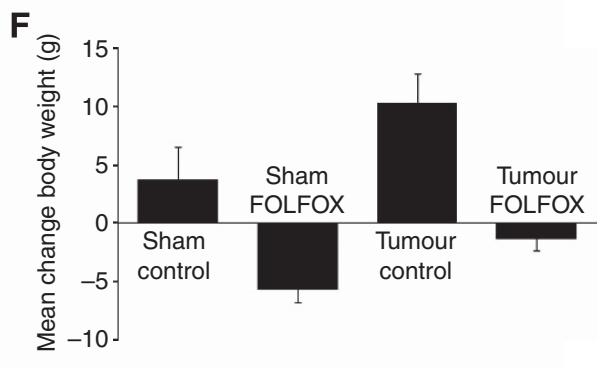

G

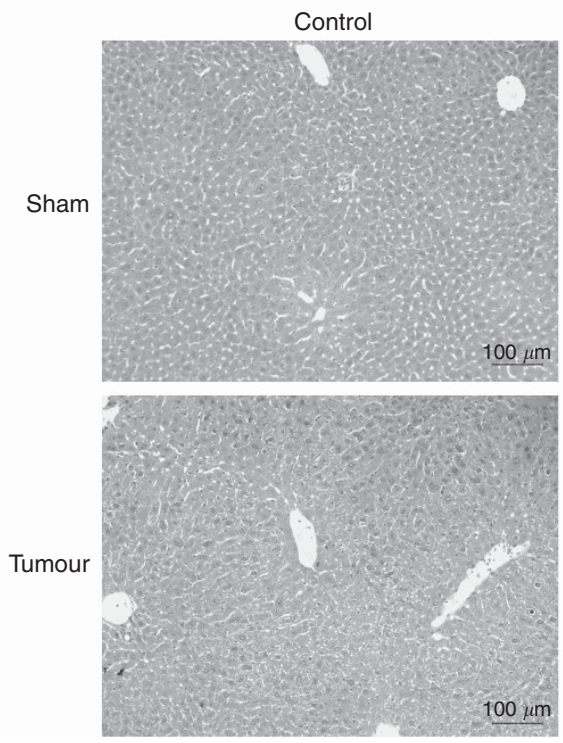

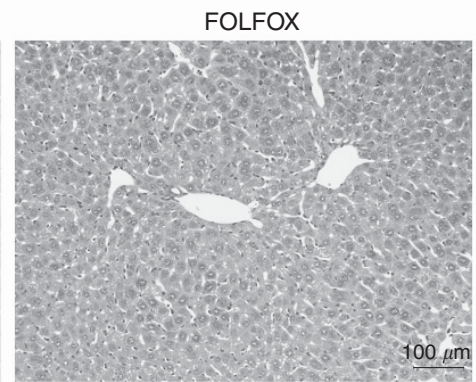

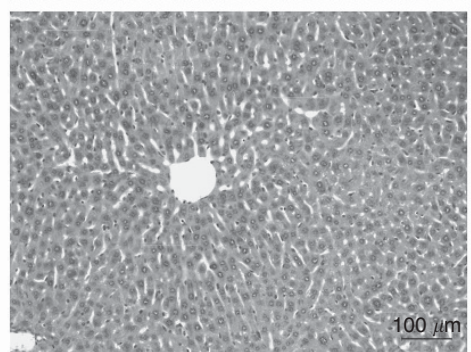

Figure 2. $\mathrm{C} 57 \mathrm{Bl} / 6$ mice underwent either laparotomy alone (shams) or laparotomy with implantation of MCA38 cells beneath the liver capsule. After 5 days tumour was detectable with IVIS imaging (A). Three weekly doses of i.p. FOLFOX resulted in a reduced tumour mass as demonstrated by tumour volume (B) and tumour/body weight ratio (C). The tumour/liver interface is shown in (D) and typical gross appearances of the tumours in (E). FOLFOX-treated animals demonstrated significant weight loss as compared with controls $(\mathbf{F})$. H\&E stained sections (G) did not demonstrate histological changes of SOS. $\left({ }^{*}=P<0.05\right)$.

of angiogenic factors, in particular, VEGF-A and VEGF-C. (Rubbia-Brandt et al, 2011; Robinson et al, 2013). The presence of tumour itself was associated with a modest increase in hepatic expression of VEGF-C (1.7-fold, $P=0.002)$, which was further increased upon treatment with FOLFOX (2.3-fold; $P=0.002)$. It is noteworthy that FOLFOX treatment alone had no effect on VEGF-C expression (Figure 3G). In contrast to this, the presence of tumour within the liver had no effect on the hepatic expression of VEGF-A. Confirming the importance of angiogenesis in the pathogenesis of SOS, we were able to demonstrate increased 
A

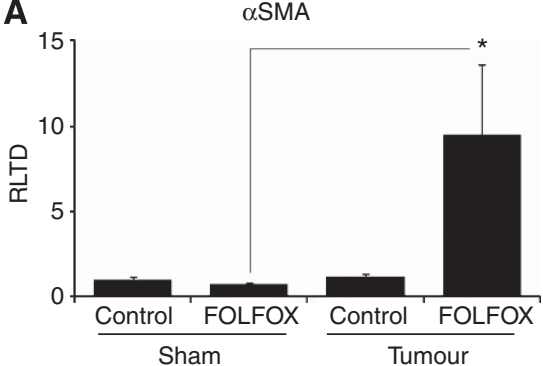

C

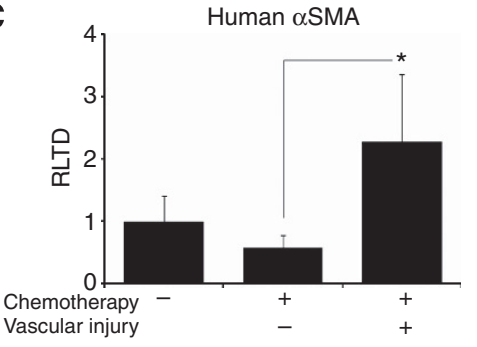

$\mathbf{F}$

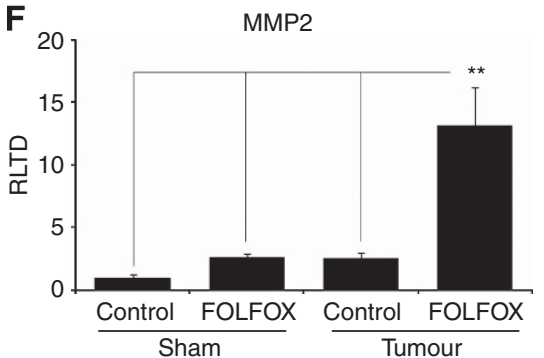

Procollagen I

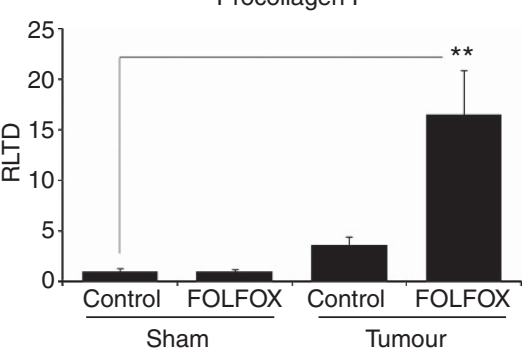

D
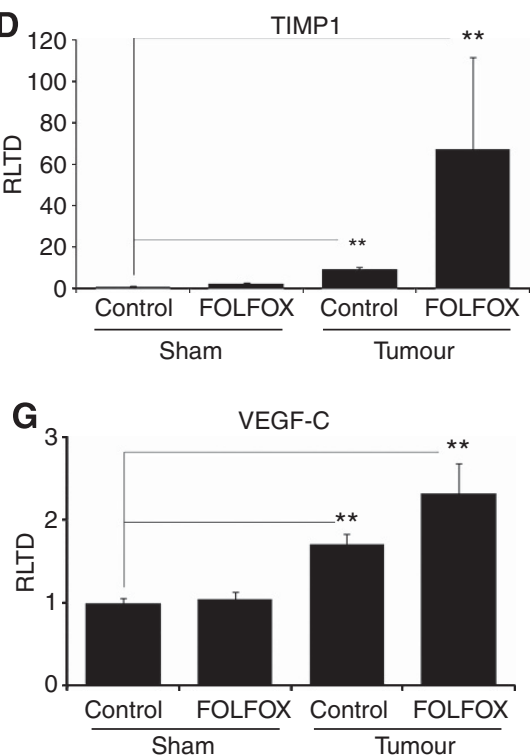

B

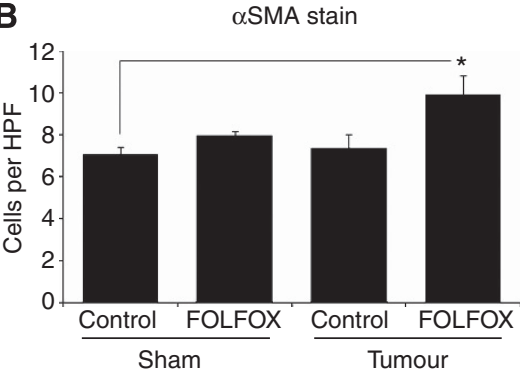

E

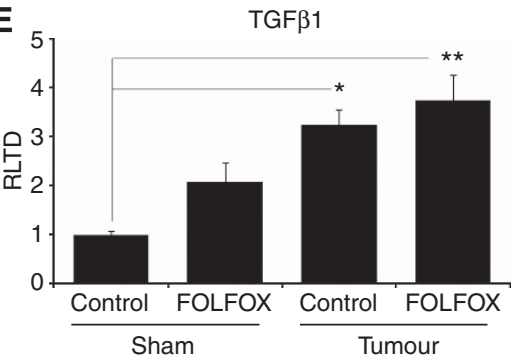

H

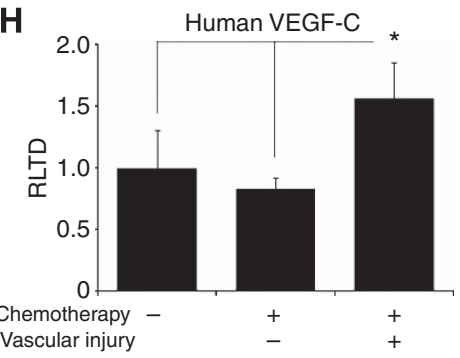

Figure 3. Tumour-bearing mice treated with FOLFOX have increased expression of the fibrogenic genes $\alpha$ SMA and pro-collagen I as compared with sham-operated vehicle controls (A). In addition, there were more $\alpha$ SMA positive cells detected by immunohistochemistry (B). Increased expression of $\alpha \mathrm{SMA}$ was also demonstrated in patients with Oxalipaltin-induced vascular injury (C). In keeping with a pro-fibrogenic environment, there is increased hepatic expression of TIMP-1 transcript in tumour-bearing FOLFOX-treated mice (D). It is likely that these changes are, in part, driven by increased expression of the master regulator TGF $\beta-1(E)$. Injury to the hepatic sinusoid in SOS is driven by gelatinases and in keeping with this there is increased expression of MMP-2 transcript in FOLFOX-treated tumour-bearing mice (F). In keeping with an angiogenic process, there is up-regulation of VEGF-C transcript in FOLFOX-treated tumour-bearing mice $(\mathbf{G})$ and in patients with Oxaliplatin-induced liver injury $(\mathbf{H}) .\left({ }^{*}=P<0.05,{ }^{\star *}=P<0.01\right)$.

expression of VEGF-C in patients with Oxaliplatin-induced vascular injury (Figure $3 \mathrm{H} ; P=0.044$ ).

Combined tumour and FOLFOX results in a prothrombotic environment. Our previous work has demonstrated that activation of the clotting cascade seems to have a role in the pathogenesis of SOS (Robinson et al, 2013). As shown in Figure 4A, treatment of tumour-bearing mice with FOLFOX is associated with a 146-fold increase in expression of the pro-thrombotic plasminogen activator inhibitor-1 (PAI-1; SERPINE1) transcript $(P=0.004)$ as well as a more modest increase in expression of von Willebrand factor $(P=0.004$; Figure $4 \mathrm{~B})$. There was a trend towards elevated vWF transcript in patients with Oxaliplatin-induced vascular injury although this did not quite reach statistical significance $(P=0.059$; Figure 4C).

In keeping with previous reports, we were able to confirm that FOLFOX administration results in the accumulation of large clusters of megakaryocytes within the spleen of both shamoperated and tumour-bearing animals treated with FOLFOX (Figure $4 \mathrm{D}$ and $\mathrm{E} ; \mathrm{P}<0.05$ ).

\section{DISCUSSION}

Until the advent of modern chemotherapy regimens, the only recognised cause of SOS was the ingestion of pyrrolizidine alkaloids. SOS was subsequently recognised as a clinical entity in patients undergoing myeloablative chemotherapy prior to bone marrow transplantation and, more recently, following administration of platinum-based chemotherapy to patients with CRLM (DeLeve et al, 2002). Until recently knowledge regarding the pathogenesis of this disease was drawn predominantly from the study of rats treated with monocrotaline which results in rapid appearance of SOS-type lesions within the liver (DeLeve et al, 1999).

Although the administration of monocrotaline may replicate many of the histological changes seen in patients with CALI, it is extremely unlikely that the stimulus driving these changes is in any way similar. To address this, we have recently established a murine model of FOLFOX-induced SOS that replicates the histological changes seen in patients receiving this chemotherapy regimen. The aim of the current study was to evaluate the potential contribution of tumour-related factors to the development of SOS. Although on this occasion, we were not able to replicate the histological features of SOS, because of the need to terminate the model with due regards to animal welfare, we have demonstrated that many of the changes in gene expression associated with the development of this condition are accelerated in tumour-bearing mice (Rubbia-Brandt et al, 2011; Robinson et al, 2013).

One criticism of the current model may be that the route of chemotherapy administration (i.p.) differs from that used in patients and therefore may limit the development of histological 
A

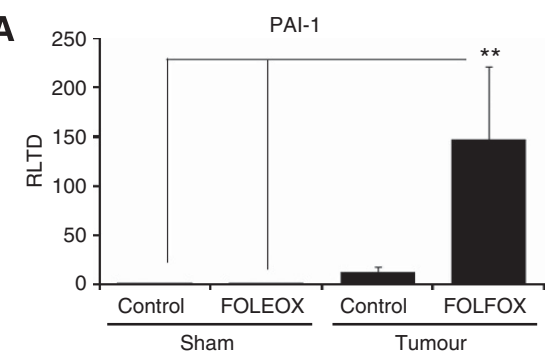

C

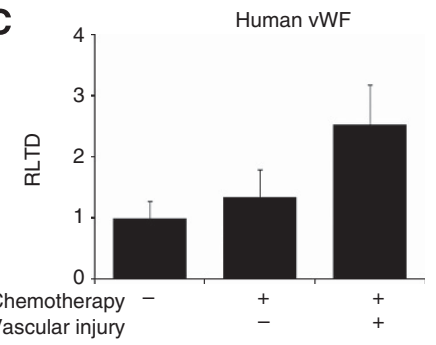

E
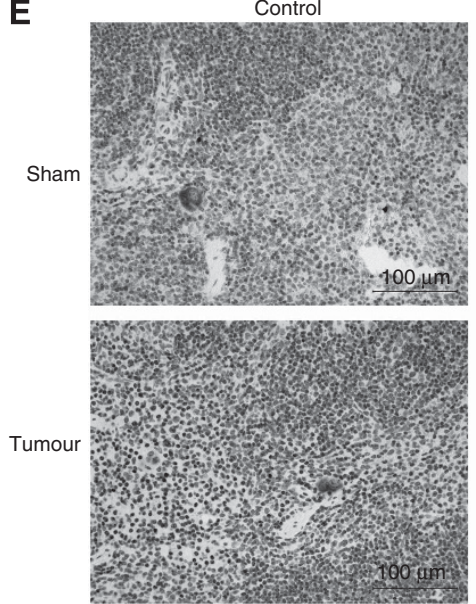

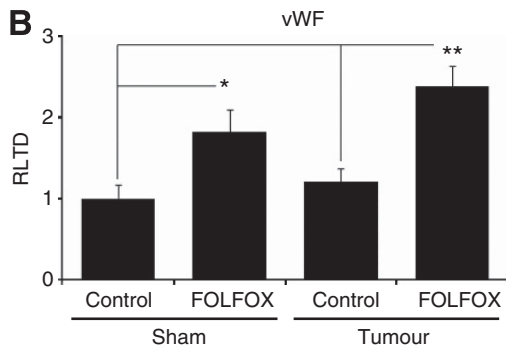

D

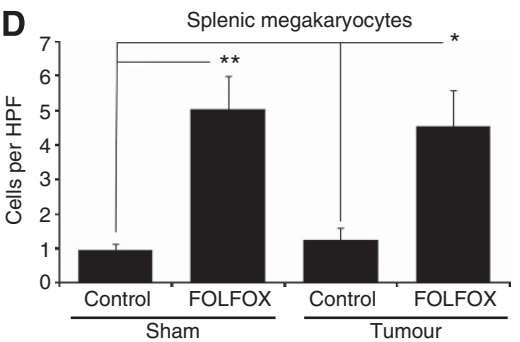

FOLFOX

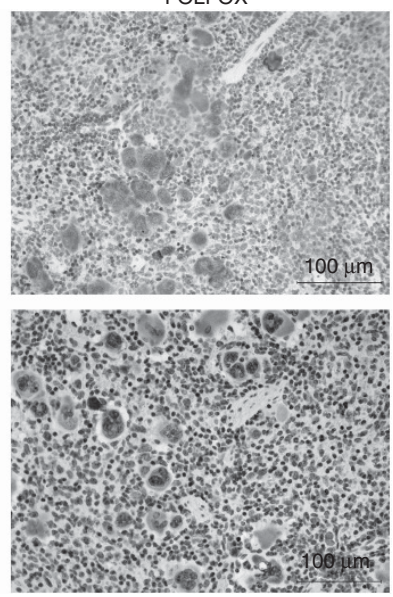

Figure 4. Oxaliplatin-induced liver injury has also been associated with a pro-thrombotic tendency in the hepatic sinusoid and in accordance with this we demonstrated increased hepatic expression of both PAI-1 (A) and vWF (B) in tumour-bearing FOLFOX-treated animals. In addition, there is a trend to increased vWF expression in patients with Oxaliplatin-induced vascular injury (C). Furthermore, there was an accumulation of megakaryocytes within the spleen ( $\mathbf{D}$ and $\mathbf{E}$ ) of these animals as determined by P-Selectin immunohistochemistry. $\left.{ }^{\star}=P<0.05,{ }^{\star \star}=P<0.01\right)$.

changes within the liver. This route was chosen as repeated intravenous administration of a compound to mice (e.g. via the tail vein) is not practical owing to the high failure rate with this technique and the need for animal restraint (Turner et al, 2011). It has been previously demonstrated that the i.p. administration of Oxaliplatin can lead to the development of neurotoxicity in mice suggesting there is significant systemic drug absorption (Ta et al, 2009). Moreover, we have recently reported that administration of i.p. FOLFOX to mice for 5 weeks does lead to the development of the histological features of SOS (Robinson et al, 2013).

Pro-thrombotic changes in the sinusoidal endothelium have been implicated in the development of SOS (Copple et al, 2002; Rubbia-Brandt et al, 2011; Robinson et al, 2013). In particular, elevated serum PAI-1 levels have been reported both in mice with FOLFOX-induced SOS as well as bone marrow transplantation patients who go on to develop SOS following myeloablative chemotherapy (Kaleelrahman et al, 2003; Robinson et al, 2013). The importance of this process is reflected in the relative success of the fibrinolytic agent defibrotide in the treatment of SOS in bone marrow transplant recipients (Benimetskaya et al, 2008). In concordance with these findings, we see increased expression of the PAI-1 and vWF genes in the liver of mice treated with FOLFOX chemotherapy, particularly in the presence of tumour.
It is likely that the presence of megakaryocytes in both the spleen and liver following FOLFOX treatment results in the release of platelets directly into the portal venous system and may contribute to the development of pro-thrombotic environment within the injured hepatic sinusoid. Given these observations, it may be that patients with particularly large tumours would benefit from treatment with antiplatelet therapy such as Aspirin which it has been suggested may reduce the incidence of SOS in Oxaliplatin-treated patients (Brouquet et al, 2009).

Despite the absence of florid fibrosis in our model, we have shown changes in gene expression in keeping with a classical fibrogenic response that we have previously reported in the context of FOLFOX-induced SOS (Robinson et al, 2013). It appears from the current study that the increase in expression of matrix remodelling genes is markedly exacerbated by the presence of tumour within the liver. It is increasingly recognised that tumour death in response to many cytotoxic agents is not a silent event but rather is associated with the production of a wide variety of mediators that are capable of producing an inflammatory response (Tesniere et al, 2008; Martins et al, 2009).

One example of how inflammatory mediator production by dying tumour cells may contribute to the development of SOS is provided by the chemokine CXCL1, the murine equivalent of IL-8, 
which is abundantly produced by FOLFOX-treated MCA38 cells and is also elevated in the serum of patients with SOS (Remberger and Ringden, 1997; Schots et al, 2003). CXCL1 exerts its effects on endothelial cells via the CXCR2 receptor with resultant increased expression of genes involved in angiogenesis such as MMP2 and VEGF (Li et al, 2003; Li et al, 2005). In addition, IL-8 signalling to human endothelial cells may increase further expression of prothrombotic genes such as PAI-1 (Cheng et al, 2008). The observation that patients with a larger tumour burden are more likely to develop SOS, despite a similar number of cycles of chemotherapy, would seem to support the concept that tumour death contributes to SOS development (Tamandl et al, 2011).

The ideal strategy for patients with CRLM receiving pre-operative FOLFOX-based chemotherapy would be to administer a drug for the duration of the chemotherapy that prevents the development of SOS. The use of antioxidants, particular those which activate the transcription factor NRF2, alongside chemotherapy has shown merit in this regard in experimental models (Wang et al, 2000; Robinson et al, 2013). It may be that this approach would be of particular value in those individuals with a large tumour burden needing prolonged treatment with pre-operative chemotherapy.

In conclusion, we have developed an experimental model which demonstrates that the development of SOS in patients with CRLM is likely to be multifactorial and supports the notion that tumourrelated factors may also contribute to this process.

\section{ACKNOWLEDGEMENTS}

This work was funded by a Wellcome Trust Clinical Research Training Fellowship (WT090974MA) awarded to Stuart Robinson. Steve White was funded by a Royal College of Surgeons (England) pump priming grant and a Spire Healthcare Project Grant. Derek Mann is supported by Wellcome Trust Grants WT084961MA and WT087961MA. The IVIS Spectrum was purchased through a Wellcome Trust Equipment Grant (WT087961).

\section{CONFLICT OF INTEREST}

The authors declare no conflicts of interest.

\section{REFERENCES}

Adam R, Bismuth H, Castaing D, Waechter F, Navarro F, Abascal A, Majno P, Engerran L (1997) Repeat hepatectomy for colorectal liver metastases. Ann Surg 225(1): 51-60; Discussion 60-62.

Adam R, Pascal G, Azoulay D, Tanaka K, Castaing D, Bismuth H (2003) Liver resection for colorectal metastases: the third hepatectomy. Ann Surg 238(6): 871-883; Discussion 883-884.

Agostini J, Benoist S, Seman M, Julie C, Imbeaud S, Letourneur F, Cagnard N, Rougier P, Brouquet A, Zucman-Rossi J, Laurent-Puig P (2012) Identification of molecular pathways involved in oxaliplatin-associated sinusoidal dilatation. J Hepatol 56(4): 869-876.

Alberts SR, Horvath WL, Sternfeld WC, Goldberg RM, Mahoney MR, Dakhil SR, Levitt R, Rowland K, Nair S, Sargent DJ, Donohue JH (2005) Oxaliplatin, fluorouracil, and leucovorin for patients with unresectable liver-only metastases from colorectal cancer: a North Central Cancer Treatment Group phase II study. Journal of Clinical Oncology 23(36): 9243-9249.

Benimetskaya L, Wu S, Voskresenskiy AM, Echart C, Zhou J, Shin J, Iacobelli M, Richardson P, Ayyanar K, Stein CA (2008) Angiogenesis alteration by defibrotide: implications for its mechanism of action in severe hepatic veno-occlusive disease. Blood 112: 4343-4352.

Boughattas NA, Levi F, Fournier C, Lemaigre G, Roulon A, Hecquet B, Mathe G, Reinberg A (1989) Circadian rhythm in toxicities and tissue uptake of 1,2-diamminocyclohexane(trans-1)oxalatoplatinum(II) in mice. Cancer Res 49(12): 3362-3368.

Brouquet A, Benoist S, Julie C, Penna C, Beauchet A, Rougier P, Nordlinger B (2009) Risk factors for chemotherapy-associated liver injuries: a multivariate analysis of a group of 146 patients with colorectal metastases. Surgery 145(4): 362-371.

Cao S, Frank C, Rustum YM (1996) Role of fluoropyrimidine schedule and $(6 \mathrm{R}, \mathrm{S})$ leucovorin dose in a preclinical animal model of colorectal carcinoma. J Natl Cancer Inst 88(7): 430-436.

Cheng M, Li Y, Wu J, Nie Y, Li L, Liu X, Charoude HN, Chen H (2008) IL-8 induces imbalances between nitric oxide and endothelin-1, and also between plasminogen activator inhibitor-1 and tissue-type plasminogen activator in cultured endothelial cells. Cytokine 41(1): 9-15.

Copple BL, Banes A, Ganey PE, Roth RA (2002) Endothelial cell injury and fibrin deposition in rat liver after monocrotaline exposure. Toxicol Sci 65(2): 309-318.

de Meijer VE, Kalish BT, Puder M, Ijzermans JN (2010) Systematic review and meta-analysis of steatosis as a risk factor in major hepatic resection. Br J Surg 97(9): 1331-1339.

DeLeve LD, McCuskey RS, Wang X, Hu L, McCuskey MK, Epstein RB, Kanel GC (1999) Characterization of a reproducible rat model of hepatic veno-occlusive disease. Hepatology 29(6): 1779-1791.

DeLeve LD, Shulman HM, McDonald GB (2002) Toxic injury to hepatic sinusoids: sinusoidal obstruction syndrome (veno-occlusive disease). Semin Liver Dis 22(1): 27-42.

Fischel JL, Formento P, Ciccolini J, Rostagno P, Etienne MC, Catalin J, Milano G (2002) Impact of the oxaliplatin-5 fluorouracil-folinic acid combination on respective intracellular determinants of drug activity. Br J Cancer 86(7): 1162-1168.

Kaleelrahman M, Eaton JD, Leeming D, Bowyer K, Taberner D, Chang J, Scarffe JH, Chopra R (2003) Role of plasminogen activator inhibitor-1 (PAI-1) levels in the diagnosis of BMT-associated hepatic veno-occlusive disease and monitoring of subsequent therapy with defibrotide (DF). Hematology 8(2): 91-95.

Keizman D, Maimon N, Ish-Shalom M, Buchbut D, Inbar M, Klein B, Bernheim J, Goldiner I, Leikin-Frenkel A, Konikoff F (2010) An animal model for chemotherapy-associated steatohepatitis and its prevention by the oral administration of fatty acid bile acid conjugate. Cancer 116(1): 251-255.

Li A, Dubey S, Varney ML, Dave BJ, Singh RK (2003) IL-8 directly enhanced endothelial cell survival, proliferation, and matrix metalloproteinases production and regulated angiogenesis. J Immunol 170(6): 3369-3376.

Li A, Varney ML, Valasek J, Godfrey M, Dave BJ, Singh RK (2005) Autocrine role of interleukin- 8 in induction of endothelial cell proliferation, survival, migration and MMP-2 production and angiogenesis. Angiogenesis 8(1): $63-71$.

Martins I, Tesniere A, Kepp O, Michaud M, Schlemmer F, Senovilla L, Seror C, Metivier D, Perfettini JL, Zitvogel L, Kroemer G (2009) Chemotherapy induces ATP release from tumor cells. Cell Cycle 8(22): 3723-3728.

Pilgrim CH, Brettingham-Moore K, Pham A, Murray W, Link E, Smith M, Usatoff V, Evans PM, Banting S, Thomson BN, Michael M, Phillips WA (2011) mRNA gene expression correlates with histologically diagnosed chemotherapy-induced hepatic injury. HPB (Oxford) 13(11): 811-816.

Rees M, Tekkis PP, Welsh FK, O'Rourke T, John TG (2008) Evaluation of long-term survival after hepatic resection for metastatic colorectal cancer: a multifactorial model of 929 patients. Ann Surg 247(1): 125-135.

Remberger M, Ringden O (1997) Serum levels of cytokines after bone marrow transplantation: increased IL-8 levels during severe veno-occlusive disease of the liver. Eur J Haematol 59(4): 254-262.

Robinson S, Manas DM, Pedley I, Mann D, White SA (2009) Systemic chemotherapy and its implications for resection of colorectal liver metastasis. Surg Oncol 20(2): 57-72.

Robinson SM, Mann J, Vasilaki A, Mathers J, Burt AD, Oakley F, White SA, Mann DA (2013) Pathogenesis of FOLFOX induced sinusoidal obstruction syndrome in a murine chemotherapy model. J Hepatol 59(2): 318-326.

Rubbia-Brandt L, Lauwers GY, Wang H, Majno PE, Tanabe K, Zhu AX, Brezault C, Soubrane O, Abdalla EK, Vauthey JN, Mentha G, Terris B (2010) Sinusoidal obstruction syndrome and nodular regenerative hyperplasia are frequent oxaliplatin-associated liver lesions and partially prevented by bevacizumab in patients with hepatic colorectal metastasis. Histopathology 56(4): 430-439. 
Rubbia-Brandt L, Tauzin S, Brezault C, Delucinge-Vivier C, Descombes P, Dousset B, Majno PE, Mentha G, Terris B (2011) Gene expression profiling provides insights into pathways of Oxaliplatin related sinusoidal obstruction syndrome in humans. Mol Cancer Ther 10(4): 687-696.

Schots R, Kaufman L, Van Riet I, Ben Othman T, De Waele M, Van Camp B, Demanet C (2003) Proinflammatory cytokines and their role in the development of major transplant-related complications in the early phase after allogeneic bone marrow transplantation. Leukemia 17(6): 1150-1156.

Soubrane O, Brouquet A, Zalinski S, Terris B, Brezault C, Mallet V, Goldwasser F, Scatton O (2010) Predicting high grade lesions of sinusoidal obstruction syndrome related to oxaliplatin-based chemotherapy for colorectal liver metastases: correlation with post-hepatectomy outcome. Ann Surg 251(3): 454-460.

Stangl R, Altendorf-Hofmann A, Charnley RM, Scheele J (1994) Factors influencing the natural history of colorectal liver metastases. Lancet 343(8910): 1405-1410.

Ta LE, Low PA, Windebank AJ (2009) Mice with cisplatin and oxaliplatininduced painful neuropathy develop distinct early responses to thermal stimuli. Mol Pain 5: 9.
Tamandl D, Klinger M, Eipeldauer S, Herberger B, Kaczirek K, Gruenberger B, Gruenberger T (2011) Sinusoidal obstruction syndrome impairs long-term outcome of colorectal liver metastases treated with resection after neoadjuvant chemotherapy. Ann Surg Oncol 18(2): 421-430.

Tesniere A, Apetoh L, Ghiringhelli F, Joza N, Panaretakis T, Kepp O, Schlemmer F, Zitvogel L, Kroemer G (2008) Immunogenic cancer cell death: a key-lock paradigm. Curr Opin Immunol 20(5): 504-511.

Turner PV, Brabb T, Pekow C, Vasbinder MA (2011) Administration of substances to laboratory animals: routes of administration and factors to consider. JAALAS 50(5): 600-613.

Wang X, Kanel GC, DeLeve LD (2000) Support of sinusoidal endothelial cell glutathione prevents hepatic veno-occlusive disease in the rat. Hepatology 31(2): 428-434.

(c) (i) (2) (2) This work is licensed under the Creative Commons By NG SA Attribution-NonCommercial-Share Alike 3.0 Unported License. To view a copy of this license, visit http://creativecommons. org/licenses/by-nc-sa/3.0/ 